\title{
电力系统电气工程自动化的智能化的应用
}

\author{
黄大立 \\ 中能祥瑞电力工程有限公司，福建 福州 350001
}

[摘要]近年来, 在多方面利好因素的影响下, 我国各个领域都得到了全面的发展进步, 在这种发展形势下, 社会的发展和民 众的生活对于电力能源的需求量在不断的增加, 这样就对我国电力系统的运行情况提出了更高的要求。电力系统其实质就是 利用输电技术、发电技术以及配电技术完成电能生产、传输的系统, 其能够将生态环境中的能源利用专业的发电装置转变为 电能, 随后传输到需要的用户。由于电源点通常都被设置在不同的区域, 所以不能加以大量的存储, 这就需要确保发电与用 电二者维持良好的平衡性。电力系统要想确保为用户提供稳定的优质电能, 那么最为重要的就是需要从各个细节入手来进行 全面的管控, 这样才能不断提升电能的使用效率。在工业生产稳步发展的影响下, 电气工程自动化整体水平随之逐渐的提升, 电气工程自动化的逐渐的成为了当前高新技术产业中的主要部分, 并被人们大范围的运用到了诸多领域之中, 取得了良好的 成绩，在推动社会经济发展方面起到了积极的影响作用。

[关键词] 智能化技术; 电力系统; 电气工程; 自动化控制

DOI: $10.33142 / h s t . v 4 i 1.3459$ 中图分类号: F407

文献标识码：A

\section{Intelligent Application of Electrical Engineering Automation in Power System}

\author{
HUANG Dali
}

Zhongneng Xiangrui Power Engineering Co., Ltd., Fuzhou, Fujian, 350001, China

\begin{abstract}
In recent years, under the influence of many favorable factors, all fields of our country have made comprehensive development and progress. In this development situation, the demand of social development and people's life for electric energy is increasing, which puts forward higher requirements for the operation of Chinese electric power system. The essence of power system is to use transmission technology, power generation technology and distribution technology to complete the production and transmission system of electric energy, which can transform the energy utilization professional power generation device in the ecological environment into electric energy and then transmit it to the required users. As the power supply points are usually set in different areas, it can not be stored in a large amount, so it is necessary to ensure a good balance between power generation and power consumption. In order to ensure the power system to provide users with stable high-quality power, then the most important thing is to start from all the details to conduct a comprehensive management and control, so as to continuously improve the efficiency of power use. Under the influence of the steady development of industrial production, the overall level of electrical engineering automation is gradually improved. Electrical engineering automation has gradually become the main part of the current high-tech industry and has been widely used in many fields. It has achieved good results and played a positive role in promoting social and economic development.
\end{abstract}

Keywords: intelligent technology; power system; electrical engineering; automatic control

引言

在当前新的历史时期中, 智能技术逐渐的转变成为了各个领域创新的主流趋势, 将智能技术合理的引用到电力系统电气 工程自动化之中, 不但可以带动电力系统运行效率的提升, 并且在促进电力行业持续健康发展方面也可以起到积极的辅助作 用。但是与其他发达国家相对比来说, 我国智能化技术水平相对较低, 不管是发展水平还是实践经验都没有达到成熟的状态, 所以在将其运用到电气工程自动化之中往往会遇到诸多的问题。要想切实的将智能化技术的优越性发挥出来, 最为重要的就是 应当充分结合各方面情况和需要来进行不断的创新, 提升智能化技术整体水平, 为电力行业的稳步持续发展创造良好的基础。

\section{1 智能化技术概述}

将智能化技术合理的运用到电力工程领域之中, 是社会发展的必然趋势, 并且在推动电力系统良好发展方面具有 重要的辅助作用。智能化技术牵涉到的层面较多, 将其合理的运用到电气工程自动化之中, 能够切实的增强整个电力 系统的智能化水平, 从以往老旧模式的人工操作转变为智能技术, 将工作人员从巨大的工作量中摆脱出来, 提升工作 的效率和效果。其次, 智能技术的大范围运用可以有效的提升各类资源的利用效率, 避免发生资源浪费的情况 ${ }^{[1]}$ 。

2 电气自动化智能控制系统在电气工程中的设计理念

2.1 集中监控设计理念

集中监控系统其实质就是利用专业的处理器将系统中所存在的各个功能加以综合处理。集中监控设计理念的主要 
核心就是保证工作的便利和高效, 系统设计模式相对较为简便, 对于控站的要求相对较低。但是其也存在诸多的问题, 因为各个功能都融入到了一个处理器之中, 所以处理器运行涉及到的工作量较为巨大, 这样就会损害到处理器运行的 整体效率。如果监控对象数量不断增加, 那么必然会加剧主机的㝋余的下降问题, 就需要安设大量的电缆, 最终会导 致整体成本的增加, 并且也会牵涉到大量的维护工作。远距离安设的电缆会受到多方面因素的影响, 这样也会对整个 系统的运行稳定性造成不良影响 ${ }^{[2]}$ 。

\section{2 远程监控设计理念}

远程监控设计能够有效的缩减使用成本, 并能够完成对电气系统的远程实时监控。但是电气工程整体线路通讯速 度相对较差, 涉及到巨大的通讯量, 所以远程监控的方法适合被运用到规模较小的电气工程系统之中。

\section{3 基于电力系统电气工程自动化的智能化应用探究}

\section{1 故障诊断以及优化设计技术的应用}

在科学技术快速发展的影响下, 大量的新型科学技术被人们研发出来, 并被运用到了诸多领域之中, 使得人们的生 活和工作发生了巨大的变化。在电力系统电气工程运行过程中, 在遇到故障之前往往都会出现诸多的征兆, 换句话说, 所有的故障都是由一定的原因导致的, 不管是外界环境因素还是人为因素, 针对各个因素加以综合分析, 我们总结出故 障自身与诸多因素之间都存在一定的关联 ${ }^{[3]}$ 。诸如: 变压器在整个电气设备中属于较为重要的一个部分, 以往针对其所实 时的检测工作和维修工作是无法高效的对其中存在的问题和安全隐患加以判断的。要想高效的对故障根源加以判断, 那 么最为重要的就是需要切实合理的运用智能化技术, 针对警告信息加以综合分析, 借助运行数据信息对整个系统加以全 面的检测, 并完成对设备的综合检测, 这样就可以更加准确的判断故障的根源, 并针对性的制定预防和解决方案, 促进 企业能够获得更加丰厚的经济和社会收益。其次, 电气设备工作的实施, 需要工作人员拥有较强的专业水平以及丰富的 实践经验, 以往电气设计工作的实施都是依赖电气设计工作人员的人为操作实现的, 这样对于整个社会的质量是无法从 根本上加以保证的, 并且还会对后续各项工作的有序开展造成一定的限制。而在针对电力系统电气工程自动化控制加以设计 的时候, 可以利用最先进的 CAD 技术以及电子计算机技术来完成设计工作，也可以结合遗传算法来针对设计加以优化完善, 这样不但切实的保证电气系统的稳定运行, 并且设计结果不管是在整体效果还是综合性能方面都能够达到最佳的状态 ${ }^{[4]}$ 。

\section{2 神经网络控制技术的应用}

神经网络控制技术拥有良好的处理综合能力, 将这项专业技术加以实践运用不但可以提升定位的效率，并且还可 以完成对电力系统电气工程自动化系统的全面把控。因为神经网络技术反转波算法具有良好的实用性, 借助神经网络 控制技术能够完成反向学习计算, 并且也能够对电气设备的运行加以全面的把控。在整个神经网络分支系统之中, 设 置了专门的配电网框架, 其作用就是针对高渗透率的能源分布起到良好的全面管控, 智能神经网络的控制效果较为良 好, 利用这项技术针对输入、输出数据进行综合管理能够实现良好的效果目标 ${ }^{[5]}$ 。

\section{3 仿真技术的应用}

仿真技术是电力电气智能化技术在电力电气工程实践运用中的一种主要表现形式, 仿真技术能够对电力系统运转 过程中所形成的各类信息数据进行全面的检测, 结合实际情况对数据加以综合分析, 最终可以切实的对电力系统的运 行状况加以了解。并且能够完成对信息的查询, 尽可能的避免信息的不准确对各项方案的制定造成一定的损害。在利 用方针技术进行信息收集的时候, 可以充分结合各方面实际情况来完成对资源的整合利用, 提升资源的利用效率, 实 现对成本的全面把控的目的。

\section{4 计算机技术的应用}

近年来, 计算机技术得到了普及和发展, 计算机技术的应用可以改变生产经营模式, 推动实现产业升级。在电力 电气工程中应用计算机技术主要可以对电力电气系统的流程进行操作, 电力电气系统智能化需要提前对电力电气系统 运行程序进行设置, 并且通过下达相应指令对电力电气系统流程进行操作。

\section{4 结束语}

总的来说, 在科学技术快速发展的影响下, 大量的新型科学技术被人们大范围的引用到了电气工程自动化之中, 智能技术的运用为电力系统的运行提供了必要的物质基础以及技术保障。在电气工程自动化的实践运用中, 企业需要 切实的结合自身各方面情况, 将企业电气化工程特征充分的展现出来, 将智能化技术的优越性彻底的施展出来, 促使 电气化工程企业的核心竞争力得以不断的提升。电气工程自动化要想确保自身未来稳定持续发展, 那么还需要相关企 业以及工作人员的协同合作。企业应当切实的引用最先进的技术设备和设计理念, 并定期组织工作人员进行专业培训, 从整体上提升工作人员的专业能力，为企业未来发展打下坚实的基础。

\section{[参考文献]}

[1] 邓刚.电力系统电气工程自动化的智能化运用 $[\mathrm{J}]$. 科技创新与应用, 2017 (15) : 193 .

[2]潘修义. 智能化技术在电力系统电气工程自动化的运用 [J]. 城市建设理论研究 (电子版), 2017 (10) : 256-257.

[3]王明伟. 基于电力系统电气工程自动化的智能化应用分析 [J].中外企业家, 2015(24): 89-90.

[4] 李龙, 陈磊, 项海波. 智能化技术在电气工程自动化中的应用 [J]. 科技风,2019(27): 97.

[5]黄奎皓. 基于电力系统电气工程自动化的智能化应用分析 $[\mathrm{J}]$. 上海大中型电机, 2020(2):20-21.

作者简介: 黄大立 (1987. $8^{-}$) 男, 毕业院校: 福建师范大学; 所学专业: 电子信息工程, 当前就职单位: 中能祥瑞 电力工程有限公司, 职务: 工程师, 职称级别: 工程师。 